ISSN: 0212-0267

DOI: http://dx.doi.org/IO.I420I/hedu2017365164

\title{
LAS LUCHAS DE PODER POR EL CONTROL DE LA UNIVERSIDAD Y DE SU FINANCIACIÓN VISTAS A PARTIR DEL LEVANTAMIENTO DE LOS ALUMNOS DEL REAL COLEGIO MÁXIMO Y SEMINARIO DE SAN LUIS DE QUITO EN $1725^{1}$
}

Power struggles for control of the University and its financing seen rising from the students of Quito's Royal College and Seminary Maximum of Saint Louis at 1725

Emiliano Gil Blanco

Universidad San Francisco de Quito (Ecuador) CESUE/ACISAL

Correo-e: emigilblanco@emilianogil.es

Recepción: 23 de abril de 20I6. Envío a informantes: 18 de junio de 2016

Aceptación definitiva: 2I de marzo de 2017

Resumen: En este estudio establecemos el origen, la economía, los enfrentamientos y la corrupción en el Real Colegio Máximo y Seminario de San Luis de Quito. Lo vamos a hacer a través del estudio de un incidente ocurrido en 1725 entre diferentes autoridades eclesiásticas y civiles por el control del Seminario y de sus finanzas. No es más que una parte del enfrentamiento entre criollos y peninsulares por el control de cargos y de los importantes ingresos que proporcionaba la Real Hacienda para financiarlo.

El presente trabajo ha sido presentado en el IX Congreso de Historia del Ecuador. Universidad Andina Simón Bolívar (UASB). Quito, is al I7 de julio de 20I5. Se circunscribe dentro del proyecto de investigación titulado La formación universitaria en Quito hasta I822. Historia de los centros educativos, sus planes pedagógicos y carrera de sus alumnos y profesores. El mismo está conformado por profesores investigadores de la Universidad San Francisco de Quito (Ecuador). 


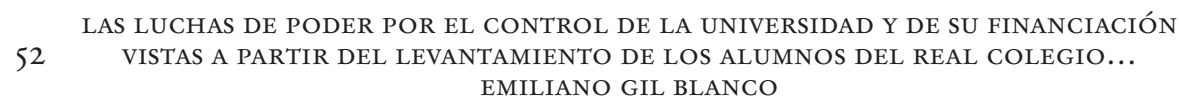

Palabras clave: Quito; Seminario San Luis; Universidad; jesuitas; criollos.

AвSTRACT: In this study we establish the origin, the economy, the confrontations and the corruption in the Quito's Royal Maximum College and Seminary of Saint Luis. We are going to do this through the study of an incident in 1725 between different ecclesiastical and civil authorities for the control of the seminary and its finances. It is only a part of the confrontation between creoles and "peninsulares» by the control of the porters and of the important incomes that provided Real Estate to finance.

KEY wORDs: Quito; University; Saint Louis Seminary; jesuits; creoles.

\section{Introducción. La educación en Quito durante la Colonia}

$\mathrm{N}$

O PODEMOS ENTRAR EN LA CUESTIÓN QUE NOS OCUPA sin hacer un breve resumen de lo que fue la historia de la educación en la Real Audiencia de Quito durante el periodo colonial hasta su independencia de España en I822. Nos vamos a centrar en Quito ya que en esta ciudad fue donde se ubicó el Seminario de San Luis. Partimos de la creación del colegio de San Juan Evangelista (I55I) y terminamos con la fundación de la Real y Pública Universidad de Santo Tomás de Aquino.

Aunque puede verse como un tema muy manido, ya que ha sido tratado por un gran número de historiadores ${ }^{2}$, han quedado muchas lagunas o vacíos en la

2 Están los estudios totales o parciales de Gil Blanco, Emiliano: «Los planes de estudio de las universidades coloniales quiteñas en el siglo xvin, hacia la reforma de la Ilustración». Capítulo iv.2, en Calderón Ortega, José Antonio; Casado Arboniés, Manuel y Díez Torres, Alejandro (coords.): Historia Universitaria de España y América, Alcalá de Henares, Servicio de Publicaciones. Universidad de Alcalá, 20I6, pp. 483-497. González Suárez, Federico: Historia eclesiástica del Ecuador, desde los tiempos de la conquista hasta nuestros días..., Quito, Imprenta del Clero, I88I. Jouanen, José: Historia de la compañía de Jesús en la antigua provincia de Quito, I570-1774, Quito, I94I-I943. KeEding, Ekkehart: Surge la nación: la ilustración en la Audiencia de Quito (I725-1812), Series: Biblioteca del Bicentenario, n. I, Quito, Ecuador, Ediciones Banco Central de Ecuador, 2005. Lucena Salmoral, Manuel: «Una universidad mayor que nunca tuvo estatutos: Santo Tomás de Quito. Funcionó con unas constituciones provisionales sin aprobación real y afrontó tres reformas estatutarias que jamás entraron en vigor», Estudios de Historia Social y Económica de América, n. ${ }^{\circ} 9$ (1992), pp. 99-II5. Malo González, Hernán (comp.): Pensamiento universitario ecuatoriano, Biblioteca Básica del Pensamiento Ecuatoriano, I4, Quito, Banco Central de Ecuador, 1980. MonCAYo DE Monge, Germania: La Universidad de Quito. Su trayectoria de tres siglos, I55I-I930, Quito, Imprenta de la Universidad Central, 1944. Rodríguez Cruz, Águeda María: Historia de las Universidades hispanoamericanas. Periodo hispánico, Bogotá, Imprenta Patriótica del Instituto Caro y Cuervo. 1973, 2 tomos. Salvador LaRA, Jorge: Historia de la Iglesia católica en el Ecuador, Quito, Ediciones Abya-Yala, 20or. Terán Najas, Rosemarie: “"La emancipada”: las primeras letras y las mujeres en el Ecuador decimonónico», Historia de la Educación, 29 (2010), pp. 35-55. VArGas, José María: Historia de la cultura ecuatoriana, Quito, Editorial Casa de la Cultura Ecuatoriana, 1965. La lista sería interminable. Hemos cuantificado 139 obras que directa o indirectamente tocan el tema de la historia de la educación y de la universidad en Quito. 
LAS LUCHAS DE PODER POR EL CONTROL DE LA UNIVERSIDAD Y DE SU FINANCIACIÓN VISTAS A PARTIR DEL LEVANTAMIENTO DE LOS ALUMNOS DEL REAL COLEGIO... EMILIANO GIL BLANCO

historia universitaria quiteña o, bien, no han sido aún bien definidos. No tenemos constancia de la existencia de una nómina de estudiantes y profesores que pasaron por sus aulas, ni de su procedencia y trayectoria posterior. Este va a ser el fin en el que se circunscribe el presente trabajo.

Como es de suponer, los primeros establecimientos educativos fundados en el Ecuador tras la conquista lo fueron en primer lugar en la ciudad de Quito. Esta obra educativa fue llevada a cabo únicamente por las diferentes órdenes religiosas establecidas en la ciudad, franciscanos, agustinos, jesuitas y dominicos.

El primer establecimiento educativo fundado fuera de Quito lo fue en la ciudad de Santiago de Guayaquil en I554, y fue erigido por los dominicos. En otras ciudades del área de la Audiencia, como Cuenca, Loja, Riobamba, Ibarra, Ambato y Latacunga, surgieron escuelas a partir de 1570 alrededor de iglesias y de conventos, donde párrocos y doctrineros catequizaron y enseñaron a leer, escribir, artes y oficios a indígenas, mestizos, criollos y españoles.

Aunque tenemos constancia de que durante la colonia se crearon escuelas particulares pagadas por los padres de familia y subsidiadas por el Cabildo, el primer colegio quiteño fue fundado por los franciscanos en I55I, el de San Juan Evangelista, más tarde llamado de San Andrés (1568)3. Estaba dedicado a la formación de indígenas para el magisterio y de mestizos que aspiraban al sacerdocio. El programa de estudios consistía en la enseñanza de aritmética, castellano, oficios como carpintería, sastrería, mecánica, y artes como pintura, dibujo o música.

La labor fundadora de los franciscanos en Quito fue continuada por los agustinos con la erección del Colegio de San Nicolás de Tolentino (I58I) y y, más tarde, por los jesuitas con la creación del Colegio Real y Seminario de San Luis (I586) y por los dominicos con la del Colegio de San Pedro Mártir (I59I) ${ }^{6}$. Estos tres últimos colegios se constituyeron como estudios generales y fueron el origen de las tres primeras universidades quiteñas: las de San Fulgencio, San Gregorio Magno y Santo Tomás de Aquino, respectivamente.

Más concretamente nos interesa el Colegio Mayor y Seminario de San Luis o Real Colegio Máximo y Seminario por su relación con los hechos que vamos a constatar más adelante. El Seminario fue fundado por fray Luis López de Solís en $1594^{7}$. Llegó a tener cincuenta becas, cuatro de origen real, veinticuatro para seminaristas y doce financiadas por otras fundaciones ${ }^{8}$. No pudo conferir grados o títulos hasta I62I, ya como Universidad de San Gregorio, por Breve del

Moncayo de Monge, Germania: op. cit., pp. 26-28.

Ibidem, p. 3 I.

Ibidem, pp. 36-42.

Ibidem, pp. 32-34.

Fraile agustino, $4 .^{\circ}$ obispo de Quito. Estudió en la Universidad de Salamanca e impartió clases en la Universidad de San Marcos de Lima, de la que fue su primer catedrático de vísperas. Rodríguez Cruz, Águeda María: op. cit., tomo i, p. 4I5. Moncayo de Monge, Germania: op. cit., p. 37. La categoría de «Máximo» se debe a que enseñaba teología y filosofía. Tuvo un gran prestigio y llegó a tener alumnos hasta de Panamá. Uzcátegui García, Emilio: Historia de la educación en Hispanoamérica, Quito, Ecuador, Ed. Universitaria, 1975, p. 292.

Moncayo de Monge, Germania: op. cit., p. 40. 


\section{LAS LUCHAS DE PODER POR EL CONTROL DE LA UNIVERSIDAD Y DE SU FINANCIACIÓN VISTAS A PARTIR DEL LEVANTAMIENTO DE LOS ALUMNOS DEL REAL COLEGIO... EMILIANO GIL BLANCO}

papa Gregorio V, títulos de bachiller, licenciado, maestro y doctor, este último a los que habían estudiado cinco años. En i8or Carlos IV procedió a su definitiva secularización?.

En 1586 se funda la primera universidad, San Fulgencio, como ya hemos apuntado, por los agustinos. En i622 se funda la segunda, San Gregorio Magno, por los jesuitas. Y en 1686 se crea la tercera, Santo Tomás de Aquino, por los dominicos. Tras la expulsión de los jesuitas de Quito en 1767 y el cierre de San Fulgencio, en I786 se refunda y seculariza la Universidad de Santo Tomás (I788), a partir de la reforma de I77I que el rey Carlos III hace de los estudios superiores en España y América, como la Real y Pública Universidad de Santo Tomás de Aquino.

Si lo contásemos de esta manera, obviaríamos una realidad histórica y esta es, en verdad, lo que realmente significaron estas fundaciones para la ciudad de Quito y para América Latina. Tengamos en cuenta que una de las primeras universidades americanas se creó en Quito, la de San Fulgencio en 1586, tras Santo Domingo, México y Lima. Pensemos que no hubo muchas ciudades durante la colonia que llegaran a tener tres universidades funcionando al mismo tiempo. Ni que tuvieran una de las bibliotecas más grandes de todo el continente, hablamos de la de la Universidad de San Gregorio Magno y del Colegio Real y Seminario de San Luis.

Estos datos nos dan a entender que en la ciudad de Quito hubo una importante actividad intelectual durante el periodo de dominación colonial.

Las tres universidades quiteñas tienen en común varias características. La primera de ellas es que su fundación surge de colegios o de un seminario-colegio: San Fulgencio a partir del Colegio de San Andrés, más tarde, de San Nicolás de Tolentino; San Gregorio Magno a partir del Seminario de San Luis; y Santo Tomás de Aquino a partir del de San Fernando. Son, por lo tanto, universidadescolegiales $^{\text {10 }}$. Otro rasgo caracterizador es que fueron fundadas por órdenes religiosas, con autorización papal y más tarde ratificadas por el rey. También todas ellas toman como ejemplo para la creación de sus estatutos los de la Universidad de San Marcos de Lima. Al ser esta última fundada a imagen y semejanza de la Universidad de Salamanca, las universidades quiteñas también tienen influencia salmantina.

La erección de universidades fue solicitada conjuntamente en 1570 por el segundo obispo de Quito, Pedro Peña; el Cabildo, y fray Pedro Bedón cuando pidieron al rey autorización para crear una universidad en la ciudad. Unos años más tarde, en I576, fue enviado a la corte fray Hernando Téllez (dominico) con el fin de defender su fundación ${ }^{\mathrm{II}}$.

Ibidem, pp. 213-218.

1o Tengamos en cuenta que hubo tres tipos de fundaciones de universidades en América: pontificias, con pase real; reales, con posterior confirmación pontificia; y conventos-universidades o colegios-universidades. Rodríguez Cruz, Águeda María: La universidad en la América hispánica, Colección Realidades, Madrid, Editorial MAPFRE, 1992, pp. 13-16.

i Moncayo de Monge, Germania: op. cit., pp. 43-45. Rodríguez Cruz, Águeda María: Historia de las Universidades hispanoamericanas, tomo I, p. 4I4. 
LAS LUCHAS DE PODER POR EL CONTROL DE LA UNIVERSIDAD Y DE SU FINANCIACIÓN VISTAS A PARTIR DEL LEVANTAMIENTO DE LOS ALUMNOS DEL REAL COLEGIO... EMILIANO GIL BLANCO

La primera fundación universitaria fue, como ya se ha expuesto, San Fulgencio por los agustinos en 1586. Los agustinos ya se encontraban establecidos en Quito desde 1573 en la parroquia de Santa Bárbara. Surgió esta a partir del Colegio de San Nicolás de Tolentino, antiguo de San Andrés de la orden franciscana. Fue autorizada a impartir grados de Artes, Teología, Derecho Canónico y cualquier otro. Concedía los títulos de Bachiller, Licenciado, Maestro y Doctor, conferibles también a otras órdenes. Su fin o caída en desgracia estuvo originada por el exceso en conferir títulos ${ }^{12}$. En 1775 se le restringió la potestad de otorgar grados a miembros de la Orden por el visitador general Joaquín Izerta. Y en I786 por Cédula del rey Carlos III fue totalmente clausurada ${ }^{13}$.

La segunda universidad quiteña por orden cronológico de fundación fue la de San Gregorio Magno. Ya en 1552 los jesuitas tenían autorizado por el papa el conferir grados ${ }^{14}$, pero no fue hasta I62I cuando lo fue para hacerlo en Quito ${ }^{15}$. Su inauguración fue en 1622 como Real y Pontificia Universidad de San Gregorio Magno. Su importancia fue grande en el desarrollo intelectual de la ciudad. Entre I622 y I650 salieron más de i60 maestros y I20 doctores. En 1759 ya hay una imprenta en la Universidad. Entre sus alumnos insignes podemos citar a Ignacio Escandón, Ignacio de Aybar y Eslaba, Marcos Alcocer, etc. Tras la expulsión de los jesuitas de Quito en $\mathbf{1 7 6 7}$, fue cancelada y su legado continuó con la Real y Pública Universidad de Santo Tomás (I786), con sus mismos estatutos, constituciones, locales, cátedras y facultades, rentas y usufructos, y parte de su profesorado ${ }^{16}$.

${ }_{12}$ La degradación de San Fulgencio fue de tal nivel que en la ciudad de Quito se hacían chanzas o burlas sobre ello, Carlos Freile cuenta al respecto: «... en la ciudad de Quito para decirle a algún pobre diablo "tonto" de manera elegante, se le lanzaba el remoquete de "Bachiller de San Fulgencio"; cuando el ofensor ansiaba llevar a la cumbre la estupidez única del enemigo le llamaba con rotundidad "Doctor de San Fulgencio". Con el paso del tiempo se redujo al "Fulgencio" llegado hasta nuestros días». Freile Granizo, Carlos: «Apodos e insultos en el Reino de Quito». Discurso de ingreso en calidad de miembro correspondiente de la Academia Ecuatoriana de la Lengua, Quito, 20I5. http://academiaec.org/?page_id=I34I.

${ }^{13}$ Bula de Sixto V (20-08-I586) de creación de la Universidad y Estatutos generales en el convento de San Agustín. Rodríguez Cruz, Águeda María: Historia de las Universidades hispanoamericanas, tomo II, pp. 482-483. Los fundadores fueron el provincial Agustín Rodríguez; los definidores Diego Mollinero, Alonso Paz y Alonso de la Fuente y Chávez, Adito Juan de Figueroa. I603 Erección de la Universidad. I602 Licencia de erección de la Universidad del Superior General de la Orden. I62I Autorización de conferir grados (Felipe III). Cátedras de Teología, Filosofía (Súmulas, Tratados de Lógica, Ánima y Metafísica). Moncayo de Monge, Germania: op. cit., pp. 45-47; Meza Cepeda, Rafael Daniel y Arrieta de Meza, Beatriz, M.: «Coexistencia de tres Universidades en el Quito Colonial (168I-1769)", Revista Venezolana de Ciencias Sociales, vol. IO, n. ${ }^{\circ}$ (2006), pp. 417-418.

${ }_{14}$ Bula de Julio III (22-IO-I552). Rodríguez Cruz, Águeda María: op. cit., tomo II, p. 34I. JouANEN, José: op. cit., pp. 569-593.

is Breve de Gregorio V (162I). Por Cédula de Felipe IV (2-02-I622) fue confirmado el Breve anterior. Rodríguez Cruz, Águeda María: op. cit., tomo II, p. 34I. Jouanen, José: op. cit., pp. 569-593.

i6 Moncayo de Monge, Germania: op. cit., pp. 47-6o; Meza Cepeda, Rafael Daniel y ArrieTA DE Meza, Beatriz, M.: op. cit., pp. 424-426. 
 \\ LAS LUCHAS DE PODER POR EL CONTROL DE LA UNIVERSIDAD Y DE SU FINANCIACIÓN VISTAS A PARTIR DEL LEVANTAMIENTO DE LOS ALUMNOS DEL REAL COLEGIO... EMILIANO GIL BLANCO}

La tercera universidad fue la de Santo Tomás de Aquino. A pesar de que fue creada en $\mathrm{I}_{68} \mathrm{I}^{17}$, no fue hasta $\mathrm{I} 686$ cuando realmente fue erigida y fundada a partir del Colegio de San Fernando. Su puesta en marcha originó un enfrentamiento con los jesuitas por la emisión de los títulos, zanjada por el rey y el papa en i740. Su plan de estudios recogía por primera vez en Quito las enseñanzas de Medicina y de Derecho Civil. Entre sus insignes estudiantes destacamos a Tomás de Jijón León, Juan Romualdo Navarro, Ramón Pérez, Nicolás Pastrana Monteserín, fray Juan de Arauz Mesía, el coronel Juan de Salinas, José Mejía y Lequerica y José Joaquín Olmedo ${ }^{18}$.

Con la reforma educativa de $\mathrm{I77}$ I de Carlos III, en $1786^{19}$ se creó la Real y Pública Universidad de Santo Tomás de Aquino que, aunque da continuidad a la del mismo nombre, no hizo sino refundir esta última con la extinta de San Gregorio, como hemos explicado anteriormente ${ }^{20}$. Con ella surge un cambio institucional, de autonomía administrativa y curricular con claras influencias de la Ilustración. Su programa académico fue muy avanzado para la época y en ella estudiaron personajes tan importantes para la historia del Ecuador como Eugenio Espejo.

Esta nueva reforma y la refundación/unificación de las universidades jesuítica y dominica no se entenderían sin el movimiento de la Ilustración, movimiento que tuvo una amplia trayectoria en Ecuador. Entre las figuras que marcaron la historia de esta última universidad cabe señalar a José Pérez Calama, obispo de Quito entre 1790 y $1792^{21}$, a Luis Héctor de Carondelet, presidente y gobernador de Quito entre 1798 y $1807^{22}$, o al mismo Eugenio Espejo.

El hecho de que las universidades fueran erigidas por órdenes religiosas y que su existencia fuera paralela durante casi un siglo (87 años) supuso una fuente de conflictos entre ellas para ver quién tenía el privilegio de emitir grados sobre las otras. También fueron motivo de enfrentamiento la distribución de los ingresos, reales y eclesiásticos, y las funciones interpuestas entre los diferentes estamentos eclesiásticos, como veremos más adelante. Tengamos en cuenta que ya desde el inicio de la conquista hubo enfrentamientos entre órdenes por el derecho a fundar universidades ${ }^{23}$.

${ }_{17}$ Breve de Inocencio XI (II-04-I68I), confirmada por decreto real de Carlos II (26-06-I683). Rodríguez Cruz, Águeda María: op. cit., tomo iI, p. 363.

i8 Moncayo de Monge, Germania: op. cit., pp. 6o-71; Meza Cepeda, Rafael Daniel y Arrieta DE Meza, Beatriz, M.: op. cit., pp. 422-424.

19 Cédula del 4 de abril de 1786 , en la que se dispuso que la Universidad se reorganizara conforme a las costumbres de las de Lima y México. Rodríguez Cruz, Águeda María: op. cit., tomo II, p. 363. Vargas, José María: La cultura de Quito colonial, Quito, Editorial Santo Domingo, 194I, p. 84.

${ }_{20}$ Moncayo de Monge, Germanía: op. cit., pp. 76-79; Meza Cepeda, Rafael Daniel, y ArrieTA DE MEZA, Beatriz, M.: op. cit., pp. 424-426.

${ }_{21}$ Lucena Salmoral, Manuel: op. cit., pp. I03-io5; Keeding, Ekkehart: op. cit., pp. 330-336

22 Larrea, Carlos Manuel: El Barón de Carondelet, XXIX Presidente de la Real Audiencia de Quito, Quito, Corporación de Estudios y Publicaciones, 1969; Lucena Salmoral, Manuel: op. cit., pp. I05-II2; KeEDing, Ekkehart: op. cit., pp. 348-349.

${ }_{23}$ Rodríguez Cruz, Águeda María: La universidad en la América Hispana, pp. 190-I9I. 
LAS LUCHAS DE PODER POR EL CONTROL DE LA UNIVERSIDAD Y DE SU FINANCIACIÓN VISTAS A PARTIR DEL LEVANTAMIENTO DE LOS ALUMNOS DEL REAL COLEGIO... EMILIANO GIL BLANCO

En el fondo no fue más que un intento de monopolizar los privilegios que tenían estas universidades para emitir grados universitarios y de limitar su importante monopolio de la educación en la ciudad.

Aquí la autoridad civil, es decir, la Audiencia y el rey, mantuvo una postura de intermediación entre las partes, hasta que a mediados del siglo xvir una Real Cédula de Felipe $\mathrm{V}$ y una bula papal decidieron la cuestión dando la razón a los jesuitas.

Por otro lado, ya a mediados del siglo xvir los agustinos acudieron al rey para intentar solventar los «agravios» que los jesuitas les causaban ${ }^{24}$ sobre repartimientos de tierras a sus conventos o misiones en el Oriente y en Quito y por la emisión de los títulos/grados universitarios. El mismo obispo de Quito fray Pedro de Oviedo salió en defensa de ambas órdenes ante el rey. Con respecto a la calidad de la enseñanza de las dos universidades se pronunció sobre ella. En la carta que remitió al rey llegó a decir que había dos universidades en Quito, una de los jesuitas, San Gregorio Magno, y otra de los agustinos, San Fulgencio, y que solamente la primera tenía una buena enseñanza ${ }^{25}$.

También hubo conflictos entre los jesuitas de la Universidad de San Gregorio y los dominicos de la de Santo Tomás de Aquino otra vez por el privilegio a graduar, pleito que terminó con el Breve de Clemente XI de $174 \mathrm{O}^{26}$, ya citado. Los jesuitas y dominicos tenían privilegios generales para graduar con validez en territorios distantes 200 millas de distancia entre sí. Con lo que resultaba antijurídica la fundación de ambas universidades, a sabiendas de que ya existía la universidad agustina de San Fulgencio.

Podían todas estas universidades impartir enseñanzas de teología, artes, retórica y gramática (Diego Rodríguez de Ocampo, 1650). Un breve del papa Inocencio XII de I693 ya concedía a los colegios jesuitas y dominicos en Santafé y Quito la facultad de conferir grados en artes, teología y derecho canónico. Breve que fue ratificado por Real Cédula en 1704, en el que también igualaba la enseñanza y grados de las universidades de jesuitas y dominicos de esas ciudades. Se establecen en las cátedras de cánones y leyes y se ordena también que se hiciesen y remitiesen al Consejo sus estatutos conforme a las Universidades de Salamanca, Lima y México.

\section{Las luchas de poder por el control del Seminario de San Luis $\mathrm{y}$ de sus ingresos. El tumulto de los alumnos de $\mathbf{1 7 2 5}$}

El conflicto más importante habido fue el ocurrido a partir del tumulto de i8 de enero de 1725 entre jesuitas, Obispado y Audiencia. Un conflicto muy peculiar

${ }^{24}$ Archivo General de Indias (en adelante AGI) Quito, 89, N. 3I. Agravios que sufren los agustinos de los jesuitas. I640-5-25. AGI QUITO, 89, N. 32. Los jesuitas se defienden de acusaciones de los agustinos. $1640-5-28$.

25 AgI Quito, 77, N. 66. El obispo sobre estado de la diócesis. 1640-5-3. Carta del obispo de Quito, Pedro de Oviedo, a S. M.

26 Rodríguez Cruz, Águeda María: op. cit., pp. I90-I9I. 
y estrambótico en el que se pueden ver las luchas de poder, los círculos de influencias e intereses, entre las entidades citadas, y la corrupción en la concesión de becas.

Pero antes hemos de tener en cuenta la posible crisis en que se encontraba el Seminario de San Luis ante la falta de ingresos. Ya Diego Ladrón de Guevara, obispo de Quito, virrey del Perú y presidente de la audiencia de Quito, comunicó al rey en I7II que el colegio no podía mantener los 24 colegiales becados que tenía. El rey respondió apremiando al obispo de Quito en el cobro de las rentas con el fin de poder mantener el colegio ${ }^{27}$.

Un informe del procurador general, Juan Bautista Mújica, desdice la posibilidad de la existencia de una crisis cuando certifica que los ingresos del Seminario eran del $3 \%$ de los ingresos de la diócesis, concretamente los ramos de mesa capitular, estipendios, capellanías y cofradías. Además, cita que se compraron en su día haciendas en Alangair, Cotocollao y otra de obrajes en San Ildefonso y que constituyeron una importante fuente de ingresos. Más a más, no todos los colegiales se beneficiaban de estos ingresos. Estaban los alumnos porcionistas o convictorios que tenían que pagar una cuota de entre 50 y 100 pesos anuales ${ }^{28}$. Si tenemos en cuenta que eran un número similar al de los becados, es decir, veinticuatro, los ingresos anuales que ingresaba el Seminario fueron entre I.200 y 2.400 pesos al año.

La Real Hacienda fue la que se encargaba de la recaudación de los impuestos anteriormente citados y la que los entregaba al obispado. Para el Colegio Seminario las cantidades recibidas en concepto de becas de la Caja de Quito ascendieron a la no despreciable suma de 21.00o pesos durante el primer cuarto del siglo xviII. Súmense estas cantidades a las abonadas por los porcionistas y estamos entonces suponiendo que mantenía un ritmo de ingresos suficiente para su continuidad.

\begin{tabular}{|r|r|}
\hline AÑO & \multicolumn{1}{|c|}{ IMPORTE $^{29}$} \\
\hline I7O3 & 500,00 \\
\hline I7O4 & 500,00 \\
\hline I7II & 500,00 \\
\hline I7I2 & 500,00 \\
\hline I7I3 & $5.006,00$ \\
\hline I7I4 & $2.878,00$ \\
\hline I7I 8 & 500,00 \\
\hline I720 & I.000,00 \\
\hline I72I & $3.664,00$ \\
\hline
\end{tabular}

27 AGI QUITO 203. Expediente sobre constituciones del colegio de San Luis y revoluciones de los colegiales años del I725 al I729. Carta de I8-IO-I7II.

28 AGI QUiTO 203. Carta del obispo de Quito al Rey de 28 de febrero de 1725.

29 Klein, Herbert S. y Tepaske, John J.: Las cartas cuentas de la Real Hacienda de la América española, siglos XVI a principios del siglo XIX, Durham, Duke University Press, 1982. La documentación manejada por los autores no muestra más que doce años de veinticinco. 
LAS LUCHAS DE PODER POR EL CONTROL DE LA UNIVERSIDAD Y DE SU FINANCIACIÓN VISTAS A PARTIR DEL LEVANTAMIENTO DE LOS ALUMNOS DEL REAL COLEGIO... EMILIANO GIL BLANCO

\begin{tabular}{|c|r|}
\hline AÑO & \multicolumn{1}{|c|}{ IMPORTE } \\
\hline I722 & $3.675,00$ \\
\hline I723 & I.9I7,OO \\
\hline I724 & 357,00 \\
\hline TOTAL & $\mathbf{2 0 . 9 9 7 , 0 0}$ \\
\hline
\end{tabular}

Para Jouanen, las causas del tumulto fueron dos. En primer lugar, la libertad de defender las nuevas ideas por parte de algunos profesores del Seminario. Nos remite a I606 y I7I5 en que el general de los jesuitas del momento hubo de tomar medidas, prohibiendo a los alumnos mantener opiniones que no fueran amparadas por la Compañía so pena de perder la cátedra ${ }^{30}$.

En segundo lugar, se refiere a los enfrentamientos nacionalistas de algunos seminaristas y profesores criollos por la preponderancia de cargos contra profesores y superiores de origen español. Los jesuitas americanos no siempre supieron mantener la disciplina y apoyaron a los alumnos criollos ${ }^{3 \mathrm{I}}$.

También expone otras causas, no tan inmediatas o graves. Una de ellas fue el enfrentamiento existente entre la Curia y el Seminario por las becas que se concedían a los seminaristas pobres. Este tema lo tratamos más adelante con la denuncia que el rector del seminario, padre Pedro de Campos, hizo en su alegato de defensa ante las autoridades ${ }^{32}$. Se admitían los becados sin la aprobación del rector. Entre estos estaban los seminaristas expulsos causantes del tumulto.

Otro frente de conflictos fue el tema de las precedencias en los actos académicos y litúrgicos que se realizaban en el seminario y en la catedral. Este tema protocolario era muy importante en toda América pues representaba la importancia o el poder que tenía cada autoridad colonial.

La causa inmediata del tumulto fue el enfrentamiento entre el obispo de Quito, Luis Francisco Romero, con el Seminario de San Luis y la Compañía de Jesús por la obligación que tenían los seminaristas de acudir forzosamente a las celebraciones ordinarias y oficios de la catedral, recogido inicialmente en las constituciones del seminario. Eran cuatro los seminaristas que acudían a los servicios ordinarios de la catedral, seis para los domingos y festivos, y ocho para las fiestas extraordinarias ${ }^{33}$. Se aumentaron en seis el número de colegiales con el fin de ayudar en los servicios de culto en días feriados de la catedral (en ese momento, dos en los ciriales, dos en los incensarios y dos en dar la paz al clero y a la ciudad y quedaban por cubrir el altar y el coro).

Los jesuitas se opusieron en todo momento a que los colegiales/seminaristas acudiesen a los oficios de la catedral aduciendo que suponía un grave relajo en la disciplina y en los estudios de los mismos. Es por ello por lo que acudieron al rey

JouAnen, José: op. cit., p. Io2.

Ibidem, p. IO2.

Ibidem, p. I02.

33 AGI QUITO 203. Carta del obispo de 4 de diciembre de 1724 sobre haberse despojado a la catedral del servicio del seminario por R.C. y pide su revocación (recibida el 2 de marzo de 1726 ). JouAnen, José: op. cit., pp. I03-IO4. 
LAS LUCHAS DE PODER POR EL CONTROL DE LA UNIVERSIDAD Y DE SU FINANCIACIÓN VISTAS A PARTIR DEL LEVANTAMIENTO DE LOS ALUMNOS DEL REAL COLEGIO... EMILIANO GIL BLANCO

con el fin de suprimir el servicio. Por Bula del papa Inocencio XIII y por Real Cédula de i de agosto de 1722, se ordenó suspender las asistencias a la catedral de los colegiales del seminario ${ }^{34}$.

En I725, el obispo Luis Francisco Romero propuso que los colegiales becados quedaran bajo el mandato de los jesuitas y los convictorios o porcionistas bajo el obispado, escisión en dos colegios con diferentes trajes, manteniendo el seminario sus haciendas, con su antigüedad y uniforme, y creando el Obispado uno nuevo partiendo de cero $^{35}$. Esta propuesta no fue tenida en cuenta.

Como consecuencia de las autorizaciones reales y papales de inasistencia de los seminaristas a la catedral, el citado obispo reaccionó suspendiendo el pago de las rentas al seminario, con el quebranto que esto supuso al mismo. Agravado por el hecho de que en aquel momento había colegiales porcionistas que no pagaban sus cuotas, dadas las diferentes procedencias de los colegiales, porcionistas y becados, siendo los primeros de origen más humilde que los segundos ${ }^{36}$.

En esta situación nos encontramos cuando el i8 de enero de 1725 se produce un tumulto entre los colegiales del seminario ${ }^{37}$. La causa estuvo en el descontento causado por la expulsión de dos colegiales teólogos, Cayetano Iglesias y Agustín Miñano, y del criado de este último, por haber acudido en julio de I724 a una corrida de toros en la plaza mayor de Quito sin el correspondiente permiso del rector del seminario.

Los estudiantes expulsos intentaron solicitar el perdón del rector, el padre Pedro de Campos, y su readmisión, aceptando el castigo que quisiera ponerles. El rector les rechazó. Los colegiales intentaron por activa y por pasiva volver a ser readmitidos por ser de fuera y de buena familia, no tener recursos y una antigüedad de seis años en el seminario.

La noche del i8 de enero de 1725 los citados seminaristas entraron en el colegio, accedieron al aposento del rector e intentaron solicitar su reingreso. El rector volvió a expulsarlos. El resto de colegiales, que estaban en la puerta de la habitación, los apoyaron, les vistieron con el hábito del seminario y fueron al refectorio. El rector siguió en su idea de expulsarles. Intentaron los colegiales la intermediación de los otros padres del colegio, mas no consiguieron su propósito.

Al día siguiente, el rector Pedro de Campos salió de visita al Colegio Máximo, ocasión que aprovecharon los colegiales para tomar el seminario e impedir su entrada, negarle obediencia y cerrar sus puertas, haciéndose fuertes en él $3^{38}$. Los colegiales se mantuvieron atrincherados durante i8 días armados hasta con un cañón que habían conseguido sacar del aledaño cuartel de la Audiencia. Para aumentar

34 AgI Quito 203. Carta del obispo de Quito al Rey de 28 de febrero de i725. Jounanen, José: op. cit., pp. I03-IO4.

35 AGI QUITO 203. Carta del obispo de Quito al Rey de 28 de febrero de 1725.

36 AgI QUITO 203. Testimonio presentado por el Padre Pedro de Campos sobre el alboroto, s/f.

37 AGI QUITO 203. Carta del obispo de Quito, Luis Francisco Romero, de 2 de febrero de 1725 a los padres jesuitas sobre el alboroto del colegio mayor y real seminario de San Luis.

38 AGI QUITO 203. Carta de 20 de febrero de I725. 
LAS LUCHAS DE PODER POR EL CONTROL DE LA UNIVERSIDAD Y DE SU FINANCIACIÓN VISTAS A PARTIR DEL LEVANTAMIENTO DE LOS ALUMNOS DEL REAL COLEGIO... EMILIANO GIL BLANCO

la fuerza de sus reivindicaciones, se pusieron en contacto con los colegiales del colegio dominico de San Fernando ${ }^{39}$.

Teniendo en cuenta que uno de los seminaristas expulsado era sobrino del obispo Romero, se puede entender el interés de este por resolver a su favor el motín. De hecho, el citado obispo se reunió con los amotinados el 29 de enero, once días después de iniciado el mismo, para ponerse de acuerdo en las acciones a realizar.

Llegados a este punto, nos damos cuenta del grado de corrupción existente en la concesión de becas del seminario. El rector Pedro de Campos llegó a hacer una relación de las que se habían concedido irregularmente. En ella consta cómo se había realizado el reparto de las becas de los colegiales de ese momento ${ }^{40}$ :

I por el obispo Sancho Andrade y Figueroa

32 por el obispo Diego Ladrón de Guevara

Io por el deán y cabildo de la catedral

8 por el deán y gobernador eclesiástico Joseph Fausto de la Cueva

I por Pedro de Zumárraga, arcediano, provisor y vicario general de la catedral.

4 por el obispo Luis Francisco Romero, entre ellos el citado Agustín Miñano, su sobrino.

Al rector no se le ocurrió otra idea que acudir a la Audiencia con el fin de solicitar la ayuda de la misma. Su presidente, Santiago Larraín, encargó al oidor Simón de Rivera acudir a solucionar el conflicto. Craso error el cometido por el citado presidente al enviar a un enemigo declarado del obispo Romero. Enterado este de las pretensiones de la autoridad civil amenazó a los jesuitas con quitarles el patronazgo del seminario por haber acudido primero a ella, en contra de las propias constituciones del colegio.

El Cabildo de la ciudad también intentó intermediar en el conflicto y propuso cambiar al rector para apaciguar los ánimos, mas no lo consiguió por la oposición de los padres jesuitas. Todas las prisas fueron pocas puesto que era menester «levantar pendones» en honor del nuevo rey Luis I. No lo consiguieron.

Las partes recurrieron oficialmente al obispo Romero, quien propuso también cambiar al rector, y volvemos a encontrarnos con la oposición de los jesuitas. No aceptaron estos los cambios hasta la llegada del provincial de su Orden. Fue entonces cuando eligieron a un nuevo rector, el padre Januario Garofalo, y con ello se apaciguó el colegio, expulsando a los principales cabecillas del levantamiento.

La Audiencia, concretamente su presidente, se justificó ante el rey diciendo que no actuó con mayor dureza por un suceso anterior en el que el obispo de Quito

39 AGI QUiTO 203. Escrito de I de febrero de 1725 del escribano público Joaquín Guerrero dando fe de los muchos testimonios sobre el alboroto.

$4^{\circ}$ AGI QUITO 203. Escrito de Is de enero de 1725 del escribano público Domingo López de Urquía a petición del padre Pedro de Campos sobre irregularidades en la concesión de las becas por el Obispado sin el preceptivo informe del rector del colegio. 
LAS LUCHAS DE PODER POR EL CONTROL DE LA UNIVERSIDAD Y DE SU FINANCIACIÓN VISTAS A PARTIR DEL LEVANTAMIENTO DE LOS ALUMNOS DEL REAL COLEGIO... EMILIANO GIL BLANCO

Sancho de Figueroa y Andrade excomulgó al oidor Fernando de Rivas por mandar encarcelar a trece colegiales del Colegio de San Luis ${ }^{41}$.

Como resultado y resolución del tumulto, el Consejo Real, con fecha 26 de mayo de 1726, respondió a la petición realizada por el fiscal de la Audiencia de Quito de 29 de marzo de 1726. En ella se ordenó que les fuera anulada la beca a los dos cabecillas del alboroto y fueran expulsados del colegio y desterrados de la ciudad por un año. Sin embargo, como había pasado tanto tiempo, propuso que, en el caso de haber sido ordenados o siendo curatas, los castigue el obispo a su arbitrio. De paso, aprovecha la ocasión para el envío de cédulas para el obispo y el presidente de la Audiencia amonestándoles por su tibieza en la resolución del conflicto y ordenarles que observen la bula papal y la Cédula Real de i722.

\section{A modo de conclusión}

Para terminar, el conflicto surgido entre el obispo de Quito, Luis Francisco Romero, y el Real Colegio y Seminario de San Luis fue producto de varias causas. Las desavenencias entre jesuitas y curia por las competencias sobre actos y celebraciones religiosas en la cátedra fue la chispa que provocó el tumulto. Detrás estuvo su financiación, las luchas de poder existentes entre criollos y españoles por los cargos eclesiásticos y civiles y la corrupción por el control de la concesión de las becas a los seminaristas.

En este concreto conflicto, las mismas continuarán y van a desaparecer con la expulsión de los jesuitas en 1767 . Todas las pertenencias, bienes e ingresos de los expulsos y de la Universidad de San Gregorio Magno y del Seminario de San Luis quedarán asignados para la nueva Real y Pública Universidad de Santo Tomás de Aquino, en verdad refundada, en 1787.

\section{Bibliografía}

Calderón Ortega, José Antonio; Casado Arboniés, Manuel y Díez Torres, Alejandro (coords.): Historia Universitaria de España y América, Alcalá de Henares, Servicio de Publicaciones, Universidad de Alcalá, 2016.

Freile Granizo, Carlos: «Apodos e insultos en el Reino de Quito». Discurso de ingreso, en calidad de miembro correspondiente de la Academia Ecuatoriana de la Lengua, Quito, 2015. http://academiaec.org/?page_id=134I.

Gil Blanco, Emiliano: «Los planes de estudio de las universidades coloniales quiteñas en el siglo xviII, hacia la reforma de la Ilustración». Capítulo iv.2, en Calderón Ortega, José Antonio; Casado Arboniés, Manuel y Díez Torres, Alejandro (coords.): Historia

${ }^{4 I} \quad$ AGI QUITO 203. Informe del presidente de la Audiencia de Quito de I2 de marzo de 1725 (recibido el 22 de junio de 1726) sobre la inquietud de los colegiales del Colegio de San Luis y remite testimonio de lo obrado. AGI QUITO 203. Informe del presidente de la Audiencia de Quito como consecuencia de la Real Cédula de 8 de junio de 1726 en que narra los acontecimientos sucedidos en el Colegio y su actuación (recibido el I7 de marzo de 1729). 
LAS LUCHAS DE PODER POR EL CONTROL DE LA UNIVERSIDAD Y DE SU FINANCIACIÓN VISTAS A PARTIR DEL LEVANTAMIENTO DE LOS ALUMNOS DEL REAL COLEGIO... EMILIANO GIL BLANCO

Universitaria de España y América, Alcalá de Henares, Servicio de Publicaciones, Universidad de Alcalá, 20I6, pp. 483-497.

González SuÁrez, Federico: Historia eclesiástica del Ecuador, desde los tiempos de la conquista hasta nuestros días..., Quito, Imprenta del Clero, i88I.

Jounanen, José: Historia de la compañía de Jesús en la antigua provincia de Quito, I570-I774. Quito, I94I-I943, Quito: http://www.cervantesvirtual.com/obra-visor-din/historia-de-lacompania-de-jesus-en-la-antigua-provincia-de-quito-I570I774-tomo-i--o/html/, I94I-I943.

KeEding, Ekkehart: Surge la nación: la ilustración en la Audiencia de Quito (I725-I8I2), Quito, Ediciones Banco Central de Ecuador, 2005.

Klein, Herbert S. y Tepaske, John J.: Las cartas cuentas de la Real Hacienda de la América española, siglos XVI a principios del siglo XIX, Durham, Duke University Press, 1982.

Larrea, Carlos Manuel: El Barón de Carondelet, XXIX Presidente de la Real Audiencia de Quito, Quito, Corporación de Estudios y Publicaciones, 1969.

Lucena Salmoral, Manuel: El Reformismo despotista en la Universidad de Quito, Cuadernos del Instituto Antonio Nebrija, Universidad Carlos III, 1999.

Lucena Salmoral, Manuel: Una universidad mayor que nunca tuvo estatutos: Santo Tomás de Quito. Funcionó con unas constituciones provisionales sin aprobación real y afrontó tres reformas estatutarias que jamás entraron en vigor. Estudios de Historia Social y Económica de América, n. ${ }^{\circ}$ (1992), 1999.

Malo González, Hernán (comp.): Pensamiento universitario ecuatoriano, Quito, Biblioteca Básica del Pensamiento Ecuatoriano, I4, Banco Central de Ecuador, I980.

Meza Cepeda, Rafael Daniel y Arrieta de Meza, Beatriz M.: «Coexistencia de tres Universidades en el Quito Colonial (168I-I769)», Revista Venezolana de Ciencias Sociales, vol. Io n. 2 (2006), pp. 415-429.

Moncayo de Monge, Germania: La Universidad de Quito. Su trayectoria de tres siglos, I55II930, Quito, Imprenta de la Universidad, I944.

Rodríguez Cruz, Águeda María: Historia de las Universidades hispanoamericanas. Periodo bispánico, Bogotá, Imprenta Patriótica del Instituto Caro y Cuervo, 1973, 2 tomos.

Rodríguez Cruz, Águeda María: La universidad en la América bispánica. Colección Realidades Americanas, Madrid, Editorial MAPFre, 1992.

Salvador Lara, Jorge: Historia de la Iglesia católica en el Ecuador, Quito, Ediciones AbyaYala, 200I.

Terán Najas, Rosemarie: “La emancipada”: las primeras letras y las mujeres en el Ecuador decimonónico», Historia de la Educación, 29 (2010), pp. 35-55.

Uzcátegui García, Emilio: Historia de la educación en Hispanoamérica, Quito, Ed. Universitaria, I975.

VARgas, José María: La cultura de Quito colonial, Quito, Editorial Santo Domingo, 194I, 269 pp.

VARgas, José María: Historia de la cultura ecuatoriana, Quito, Editorial Casa de la Cultura Ecuatoriana, 1965 .

\section{Referencias documentales}

Archivo General de Indias (Sevilla, España)

- AgI Quito, 77, n. ${ }^{\circ}$ 66. El obispo sobre estado de la diócesis. I640-5-3. Carta del obispo de Quito, Pedro de Oviedo, a S. M.; dice que las órdenes religiosas viven con compostura; pondera los inconvenientes que tienen las doctrinas; dice que hay dos universidades en Quito una de los jesuitas y otra de los agustinos y que solamente la primera tiene una buena enseñanza; cuenta el traslado sin permiso de las monjas de la Concepción a su nuevo convento y cómo se solucionó. 
LAS LUCHAS DE PODER POR EL CONTROL DE LA UNIVERSIDAD Y DE SU FINANCIACIÓN VISTAS A PARTIR DEL LEVANTAMIENTO DE LOS ALUMNOS DEL REAL COLEGIO... EMILIANO GIL BLANCO

- Agi Quito, 89, n. ${ }^{\circ}$ 3I. Agravios que sufren los agustinos de los jesuitas. I640-5-25. Carta de los religiosos de la orden de San Agustín de Quito a S. M. dando cuenta de los agravios que sufren de los religiosos de la Compañía de Jesús que les ocupan sus tierras.

- AGI QUiTo, 89, n. ${ }^{\circ}$ 32. Los jesuitas se defienden de acusaciones de los agustinos. I640-5-28. Carta de los religiosos de la Compañía de Jesús a S. M. defendiéndose de las acusaciones de los agustinos.

- AGI QUiTo 203. Expediente sobre constituciones del colegio de San Luis y revoluciones de los colegiales años del I725 al I729. 